\title{
Peristomale Wunden - Ursachen und Behandlung
}

\author{
Nahtdehiszenz, Abszess, Mazeration Komplikationen am Stoma direkt als \\ auch der peristomalen Haut stellen die Betroffenen und die Pflege immer \\ wieder vor neue Herausforderungen. Im Folgenden ein Überblick über einzelne \\ peristomale Komplikationen, Ursachen und Versorgungsmöglichkeiten.
}

I

n Deutschland gibt es zurzeit ca. 160.000 Stomaträger - mit zunehmender Tendenz. Auch in den nächsten Jahren werden weiter steigende Zahlen erwartet, die sich durch verbesserte OPTechniken, bessere medizinische Versorgung und eine erhöhte Lebenserwartung erklären lassen. Es gibt verschiedene Gründe, warum Stomata angelegt werden, wie chronische Darmerkrankungen, Karzinome sowie ein vorübergehender Anastomosenschutz. Entsprechend unterscheiden sich die Anlagen in protektive - vorübergehende - Anlagen, die die im Darm angelegte Nahtstelle schützen sowie in permanente - auf Dauer angelegte - Stomata. Eine weitere Unterscheidung der Stomaanlagen richtet sich nach der Stomaart, d.h. welcher Teil des Darms (oder der Blase) betroffen ist. Hierzu gehören Colostomien (Dickdarm), Ileostomien (Dünndarm) und Urostomien (künstliche Harnableitungen).

\section{Studie zu Selbstversorgungskompetenz}

Bei einer so hohen Zahl von - zum Teil auch nur kurzfristig - Betroffenen stellt sich die Frage, wie gut die Erkrankten im Alltag mit der Situation klarkommen. Eine Studie der beiden Selbsthilfeorganisationen Deutsche ILCO e.V. und Stoma-Welt und der Fachgesellschaft Stoma, Kontinenz und Wunde (FgSKW) „Zufriedenheit und Selbstversorgungskompetenz" hat ergeben, dass 85\% der 511 Teilnehmer (Stomaträger im Alter zwischen 23 Tagen und 43 Jahren sowie Angehörige) ihre Stomaanlage selbstständig versorgen und damit eine hohe Autonomie besitzen. 11\% benötigen bei der Stomaversorgung Hilfe durch Angehörige, die restlichen 4\% durch einen Pflegedienst. 86\% der Befragten verfügten ihrer Meinung nach über ein gutes bis sehr gutes Wissen bezüglich ihres Stoma, der Stomapflege und der Versorgung und die dazu benötigten Hilfsmittel. 70\% konnten auftretende Komplikationen gut einschätzen und sich selber helfen beziehungsweise Hilfe einfordern. Hierbei zeigte sich, dass je älter die Stomaanlage, desto höher war auch die Versorgungskompetenz der Betroffenen. Komplikationen am und um das Stoma treten relativ häufig auf, in der Befragung gaben 77\% an, in den letzten drei Monaten Komplikationen gehabt zu haben. Ein Zusammenhang zwischen dem Alter der Stomaanlage oder der Versorgungskompetenz Betroffener war hierbei nicht zu erkennen.

\section{Vielfältige peristomale Hautläsionen}

Was also genau sind Stomakomplikationen und wie entstehen sie? Die Literatur bezieht sich hierbei auf alle Hautirritationen der peristomalen Haut, unabhängig von Ursache, Ausdehnung, Zustand des Gewebes und der Tiefe der Schädigung. Zu den peristomalen Wunden gehören:

- Nahtdehiszenz

- Abszess

- Mazeration

- Toxisches Kontaktekzem

- Allergie

- Keimbefall (Mykose, MRSA)

- Chronische Hautfeuchtigkeit

- Pseudoepitheliomatosis

- Hyperkeratose

- Follikulitis

- Enterocutane Fistel

\section{Ursachenanalyse und zielgerichtete Behandlung}

Im Folgenden zu den einzelnen peristomalen Komplikationen, ihre Ursachen und Lösungsvorschläge bezüglich der Behandlung:

Nahtdehiszenz: Bei einer Nahtdehiszenz sind die Nähte, mit denen das Stoma an der Bauchdecke fixiert wurde, zum Teil oder ganz ausgerissen (Abb. 1). Der Grund hierfür liegt darin, dass zu viel Zug auf dem freipräparierten Darm herrscht. Bei der Behandlung der Nahtdehiszenz muss unbedingt darauf geachtet werden, dass die Ausrissstellen vor den aggressiven Ausscheidungen geschützt und gut abgedeckt werden, gegebenenfalls mit Paste, Hautschutzringen oder Modellierstreifen. Bei stark nässenden Wunden kann zusätzlich mit einer Alginateinlage gearbeitet werden.

Abszess: Bei einem Abszess findet man die üblichen Zeichen wie Rötung, Schwellung, Schmerz und erhöhte Temperatur im Bereich der peristomalen Haut. Für eine erfolgreiche Therapie ist eine Abzessspaltung unerlässlich, bei Bedarf können auch hier zusätzliche Wundversorgungsmaterialien eingesetzt werden. 
Mazeration: Es liegt eine Entzündung der peristomalen Haut vor, mit unterschiedlichen Ursachen und Schweregraden. Sie reichen von Rötung (Hautirritation als Vorstufe) bis hin zu nässenden Hautdefekten. Ursachen hierfür können Allergien, Pflegefehler, zu lange Tragezeiten des Hautschutzes oder auch zu große Ausschnitte in der Stomaplatte sein. Deshalb steht bei der Behandlung die Ursachenforschung an erster Stelle. Eine gute Abdichtung der Stomaversorgung ist unerlässlich, ebenso der Einsatz von hygroskopischen Hautschutzmaterialien.

\section{- Je älter die Stomaanlage, desto höher auch die Versorgungskompetenz der Betroffenen.}

Toxisches Kontaktekzem: Ursachen können vielfältig sein, z.B. undichte Versorgungen oder zu große Ausschnitte der Versorgung und dadurch Reizung und Verletzung der Haut durch den Kontakt mit der Ausscheidung. Mechanische Verletzung der Haut beim Entfernen der Stomaplatte sowie falsche Reinigungsmittel beziehungsweise Hautpflege ebenso. Bei einem toxischen Kontaktekzem ist die stomaumgebende Haut gerötet, feucht und glänzend (Abb. 2). Die Betroffenen beklagen ein Jucken und Brennen, man findet häufig den Verlust der obersten Epidermis. Für die Behandlung wird hier zuerst eine gut abdichtende Versorgung gewählt, ebenso die Ausschnittgröße der Stomaversorgung mit Hilfe einer Messschablone angepasst. Stomahesives Adhäsivpulver bindet die Feuchtigkeit der nässenden Haut und verstärkt die Haftkraft der Stomaplatte. Ein Hautschutzfilm auf wässriger Basis schützt die Haut vor den Ausscheidungen, eine zusätzliche Abdichtung mit Ringen, Modellierstreifen oder Paste rundet die Versorgung ab. Falls nötig, können auch zusätzliche Wundversorgungsprodukte eingesetzt werden.

Allergie: Eine Allergie äußert sich durch gerötete, juckende und teilweise auch nässende Haut. Im fortgeschrittenen Stadium findet
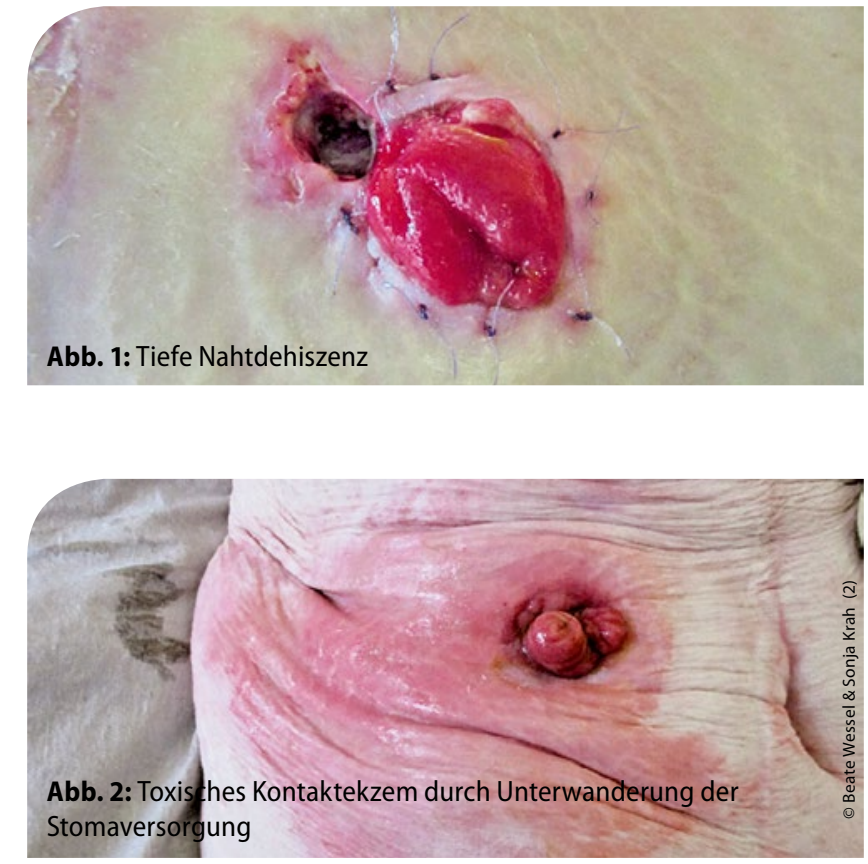

man außerdem Bläschen, Papeln und auch manchmal weißlichgelbe Beläge. Eine Allergie ist in ihrer Ausprägung meistens scharf begrenzt. Ursache hierfür ist eine Überempfindlichkeit gegen Pflege- oder Versorgungsartikel. Deshalb sollte zuallererst eine Testung des Allergieauslösers erfolgen, hierbei liegt der Fokus auf dem Pflasterrand der Stomaplatte, dem Hautschutz, aber auch auf Seife oder anderen Pflegemitteln, die benutzt werden. Bei Allergikern kann man auch schon prae-operativ eine solche Testung durchführen, um eventuelle Risikofaktoren frühzeitig auszuschließen. Sollte der Auslöser in der Zusammensetzung der Stomaplatte zu finden sein, muss auf ein anderes Versorgungssystem umgestellt werden.

\section{Hier steht eine Anzeige.}


Keimbefall: Bei Mykose oder MRSA treten auf der stomaumgebenden Haut ähnliche Symptome auf. Das Erscheinungsbild ist hier aber eher diffus. Auslöser ist wie bei einer Allergie ein Befall von Candida oder anderen Keimen (z.B. MRSA). Ein reduzierter Allgemeinzustand des Betroffenen, lange Antibiotikaeinnahme oder ein bereits vorhandener Soor im Magen-Darm-Trakt sowie mangelnde Stomahygiene können Gründe sein. Um den Erreger zu kennen und richtig zu behandeln, muss als erstes ein Abstrich der betroffenen Haut erfolgen. Je nach Erreger wird dann lokal ein wässriges, nicht fettendes Antimykotikum oder eine Desinfektionslösung angewendet. Bei einem ausgeprägten Keimbefall kann zusätzlich eine systemische Behandlung gewählt werden. Eine gute Stomahygiene ist unerlässlich.

Chronische Hautfeuchtigkeit: Hier findet man weiße, aufgeweichte, schmerzhafte, schrumpelige Hautstellen auf der peristomalen Haut, stecknadel- bis linsengroße Gewebsgranulationen. Die Ursachen liegen in einer andauernden Feuchtigkeit unter der Versorgung (tritt vermehrt bei Urostomien auf). Die Gründe können ein zu großer Lochausschnitt der Platte oder zu lange Wechselintervalle sein - je nachdem wird also der Lochausschnitt angepasst und das Wechselintervall verkürzt. Stomahesives Adhäsiv-Puder, ein zusätzliches Abdichten mit Ringen oder Paste sowie eine kurzzeitige Verwendung von zusätzlichen Hautschutzplatten kann notwendig sein. Die Pseudoepitheliomatosis ist eine unbehandelte Form der Chronischen Hautfeuchtigkeit, mit überschießender Granulation und Warzen- / Polypenbildung. Zusätzlicher Druck durch convexe Versorgung und Gürtel wirkt dem entgegen.

Hyperkeratose: Als peristomale Komplikation äußert sich die Hyperkeratose durch eine weißliche Verfärbung der Haut mit Knötchenbildung; als Ursache liegt eine übermäßige Verbreiterung der Hornhaut durch vermehrte mechanische Belastung vor. Zur Behandlung muss die Versorgung abgedichtet, ebenso sollte auch hier zusätzlicher Druck, beispielsweise durch convexe Platten/Gürtel ausgeübt werden.

Follikulitis: Bei einer Follikulitis entzünden sich die Haarkanäle in der Umgebung der Stomaanlage. Ursachen sind das Herausreißen der Haare beim Versorgungswechsel, die Haarkanäle werden freigelegt und es erfolgt eine Verschmutzung mit Stuhlkeimen. Der Stomabereich muss also zuerst gründlich rasiert werden. Hierbei sollten Einmalrasierer benutzt und die empfindliche Stomaschleimhaut vor dem scharfen Rasierer mit Hilfe einer Kompresse geschützt werden. Dann wird die gereizte Haut mit einer Hautschutzplatte abgedeckt. Kurzfristig sollte auf ein zweiteiliges Versorgungssystem umgestellt werden, damit die Stomaplatte nicht zu häufig gewechselt werden muss und keine weiteren Hautreizungen entstehen.

Enterocutane Fistel: Eine enterocutane Fistel ist eine epithelisierte Verbindung zwischen Darm und Haut und fördert Stuhlgang und Sekret. Bei der Versorgung ist darauf zu achten, dass die Fistel freigehalten wird und sich in den Beutel entleeren kann. Die Umgebungshaut ist mit einer Stomaplatte zu schützen und kann zusätzlich mit Stomapaste abgedichtet werden.

\section{Ursachenforschung entscheidend für richtige Behandlung}

Peristomale Hautläsionen sind sehr vielfältig und ihrer Entstehung liegen oft unterschiedliche Ursachen zugrunde. Eine genaue Ursachenforschung ist deshalb für die richtige Behandlung enorm wichtig: War die Haut vor dem Aufkleben der Versorgung trocken und fettfrei? Wurden Pflasterlöser oder andere Zusätze benutzt - und diese gegebenenfalls auch wieder rückstandslos entfernt? Wurde die Versorgung nach dem Anbringen „,angewärmt" und hatte Zeit und Ruhe, gut zu haften? Liegt das Stoma an einer anatomisch schwierigen Stelle (Falte, Knochenvorsprünge)? Wurde die Tragezeit überschritten? Wurde die Lochgröße angepasst?

Regelmäßiger Kontakt mit einer Stomafachkraft beziehungsweise einem Pflegeexperten unterstützt den Betroffenen, sein Stoma fachgerecht zu versorgen, Komplikationen frühzeitig zu erkennen und adäquat zu behandeln.

>II

\section{Literatur bei der Verfasserin}

\section{PFLEge EINFACH MACHEN}

Komplikationen am und um das Stoma treten relativ häufig auf, in der Befragung von Stomaträgern gaben $77 \%$ an, in den letzten drei Monaten Komplikationen gehabt zu haben.

Die Ursachen sind vielfältig, eine genaue Analyse, ob es sich beispielsweise um eine Nahtdehiszenz, einen Abszess, eine Mazeration oder eine Follikulitis handelt, ist für eine zielgerichtete Therapie und Versorgungsanpassung notwendig.

Empfehlenswert ist regelmäßiger Kontakt zu Stomafachkräften / Pflegexeperten, um deren Expertise zu nutzen.

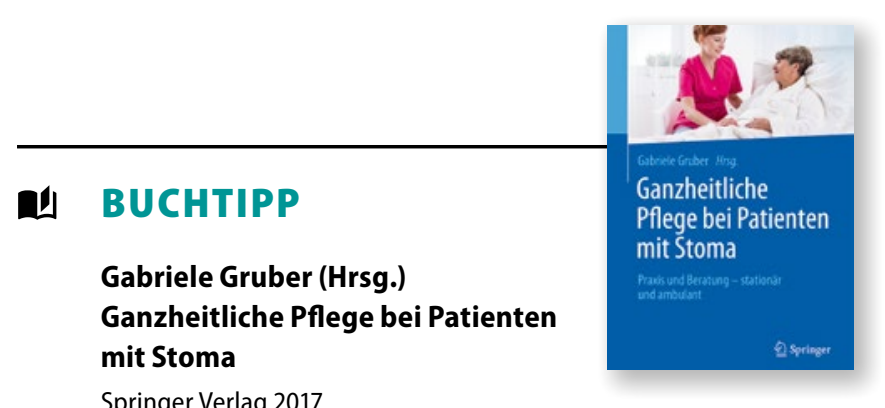

ISBN 978-3-662-48428-9; 43,73€

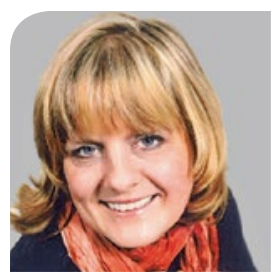

\section{Ute Marienfeld}

Pflegeexpertin Stoma, Kontinenz, Wunde Vorstand Fachgesellschaft Stoma, Kontinenz und Wunde (FgSKW) Ute.Marienfeld@fgskw.org 


\section{Hier steht eine Anzeige.}

算 Springer 